\section{Avaliação de satisfação de usuários: considerações teórico-conceituais}

\author{
User satisfaction assessment: \\ theoretical and conceptual concerns
}

\begin{abstract}
1 Instituto de Saúde Coletiva Universidade Federal da Bahia, Salvador, Brasil.

Correspondência M. A. Espiridião Instituto de Saúde Coletiva, Universidade Federal da Bahia. Rua Almirante Barroso 193, apto. 306, Salvador, BA 41950-350, Brasil. moniqueesper@yahoo.com.br
\end{abstract}

\section{Abstract}

The present article develops a critical analysis of the scientific output on user satisfaction in health services, focusing specifically on theoretical and conceptual aspects. The article discusses the understanding of satisfaction as a concept and its theoretical references and determinants and application of the concept to the field of services. A total of 56 articles were analyzed, published mainly in periodicals indexed in MEDLINE and Web of Science from 1970 to 2005. The concepts identified in this literature review come mainly from marketing and social psychology, emphasizing the theory of attitude, fulfillment of expectations or needs, and equity. There is a loss of analytical usefulness in the concept of satisfaction insofar as it is extended to various dimensions of health services.

Consumer Satisfaction; Services Evaluation; Quality
Monique Azevedo Esperidião ${ }^{1}$ Leny Alves Bomfim Trad ${ }^{1}$

\section{Introdução}

De acordo com Chen 1, a literatura sobre avaliação, durante muito tempo, desprezou a importância da teoria no processo avaliativo, constituindo-se em modelos de avaliação do tipo "caixa preta”, nos quais os resultados são produzidos sem a explicitação de referenciais teóricos ou metodológicos consistentes. Deslandes 2 reflete que, embora a avaliação de serviços atualmente lance mão de teorias e metodologias, geralmente oriundas das ciências sociais, em sua prática institucional a dimensão técnicooperacional da avaliação é reificada de tal modo, que a discussão teórico-metodológica se torna obscurecida.

O sentido fundamental ao se teorizar no campo da avaliação dos serviços de saúde é, sobretudo, o de buscar converter os conceitos em estratégias, critérios e padrões de medição, a fim de contribuir para a produção de medidas úteis que auxiliem na tomada de decisão e subsidiem aperfeiçoamentos no âmbito dos serviços. A utilização dos conceitos se torna importante na medida em que permitem representar aspectos da realidade, através de construções lógicas estabelecidas de acordo com um quadro teórico de referência.

De acordo com Mendonça 3, todo conceito possui uma conotação ou compreensão e, pelo menos uma denotação, extensão ou domínio de aplicação. A compreensão de um conceito é 
o conjunto das propriedades e relações que o definem. A denotação é o conjunto de todos os objetos aos quais se pode aplicar o conceito. Conotação e denotação se comportam inversamente: quanto mais limitada for a compreensão de um conceito, mais amplo será o seu domínio de aplicação e vice-versa ${ }^{3}$. No processo de comunicação, os conceitos se tornam manifestos por meio de termos ou palavras; daí que um conceito requer que se utilize o termo mais adequado, capaz de expressar através de seu significado o que realmente ocorre na realidade empírica.

A polissemia no campo da avaliação em saúde é fenômeno retratado por diversos autores da área. No caso das avaliações de satisfação de usuários de serviços de saúde, reflete-se que, embora o termo "satisfação" esteja na ordem do dia, sendo referido em larga escala na literatura internacional, é um conceito cujos contornos se mostram vagos, reunindo realidades múltiplas e diversas.

Os estudos de satisfação de usuários ganharam destaque na literatura principalmente na década de 1970, nos Estados Unidos e na Inglaterra, inseridos no paradigma do consumismo e na cultura da qualidade 4,5 . Neste contexto, a satisfação do usuário é considerada uma meta a ser alcançada pelos serviços, devendo, portanto, ser pesquisada visando a aperfeiçoamentos no sistema de serviços de saúde. No Brasil, os estudos se desenvolveram na década de 1990, a partir do fortalecimento do controle social no âmbito do SUS, por intermédio da participação da comunidade nos processos de planejamento e avaliação.

O interesse pela temática foi reforçado também porque estudos mostraram que usuários satisfeitos tendem a aderir ao tratamento prescrito, a fornecer informações importantes para o provedor e a continuar utilizando os serviços de saúde 6,7,8,9,10,11,12, sendo referido, ainda, que pacientes satisfeitos são mais propensos a ter melhor qualidade de vida 7 .

Partindo do pressuposto de que a temática de satisfação de usuários apresenta-se em um cenário de polissemia conceitual e escassa teorização, este artigo analisa o conceito de satisfação empregado nas avaliações de satisfação de usuários de serviços de saúde, sistematizando o conjunto de críticas presentes na literatura, com base em três dimensões principais: (i) a compreensão do conceito de satisfação e seus quadros teóricos de referência; (ii) os determinantes da satisfação; (iii) a aplicação do conceito de satisfação no âmbito dos serviços de saúde.

Foram analisados 56 artigos publicados em revistas indexadas pelo MEDLINE e Web of
Science no período de 1970 a 2005. Pesquisas manuais também foram conduzidas valendose de listas de referências de outras revisões, buscando acessar os artigos considerados importantes. Utilizaram-se como descritores principais as palavras-chaves: patient satisfaction, evaluation, health services, user satisfation, theories, concepts, review, combinadas de diferentes formas.

\section{Polissemia e fragilidade conceitual nos estudos de satisfação}

Inicialmente, vale destacar que há na literatura da avaliação de serviços de saúde pelo usuário uma vasta terminologia para designar o conceito de usuário: patient, consumer, client, costumer e user, com predomínio do primeiro, que usualmente carrega certa noção de "passividade". Os termos client, costumer e consumer são oriundos do setor privado 13 e refletem a percepção de que o papel do usuário na arena dos serviços de saúde deve ser similar ao papel que exercem quando compram produtos e serviços no mercado ${ }^{9}$. Consumer encontra-se mais relacionado à idéia de coletividade, enquanto costumer refere-se ao indivíduo 13 . O termo "cliente" (client) designa mais explicitamente aquele que paga pelo serviço 14 .

O termo "usuário" (user), por sua vez, é pouco utilizado em âmbito internacional, diferentemente do que acontece no Brasil, onde é empregado na literatura científica e nos documentos oficiais. A utilização desse tem como pano de fundo a assunção de que o sujeito que usa os serviços de saúde está para além de um paciente ou de consumidor, visto que especificidades colocadas no campo da saúde necessariamente incorporam noções de cidadania, pressuposta pela idéia de direito social 15 .

Quanto ao conceito de satisfação de usuários, a grande maioria dos artigos pesquisados 4,13, $16,17,18,19,20,21,22,23,24,25,26,27,28$ apresenta a crítica de que as avaliações de satisfação de usuário não são capazes de fornecer um modelo teórico razoavelmente consistente que possa dar conta de sua complexidade. Em termos mais específicos, alguns autores 5,6,29,30 criticam o predomínio de estudos de base empírica. Linder-Peltz ${ }^{5}$ questiona também o fato de que poucas pesquisas buscaram testar hipóteses ou teorias (theory-testing studies), ou mesmo construir uma teoria (theory-building studies). 


\section{Compreensão do conceito de satisfação de usuários: modelos oferecidos pela literatura}

A revisão bibliográfica de Ware \& Snyder 26 identificou que, até 1975, não havia uma formulação do conceito de satisfação de usuário. Segundo Linder-Pelz 5, os primeiros estudos publicados sobre a categoria satisfação focalizavam a satisfação com o trabalho ou emprego (job satisfaction), dos quais derivaram-se considerações acerca da satisfação do paciente.

Trinta anos depois da primeira revisão realizada por Ware \& Snyder 26, a revisão de Turris 4 ratifica que há um largo consenso na literatura em considerar que a satisfação do paciente é um conceito pouco teorizado.

Para esse autor 4 , os conceitos atualmente oferecidos nos permitem apenas construir uma abordagem limitada do tipo checklist para a obtenção da satisfação do paciente, no lugar de desenvolver uma compreensão de questões mais amplas sobre as experiências individuais relativas aos serviços de saúde. O autor destaca a necessidade do uso de diferentes lentes teóricas para o entendimento da satisfação de pacientes, considerando diferentes valores, crenças e visões de mundo, sem o que os pesquisadores continuarão selecionando os indicadores "mais óbvios” para a mensuração da satisfação.

Os enfoques teóricos encontrados para a compreensão da satisfação de usuários de serviços de saúde são predominantemente abordagens da escola de psicologia social, de vertente marcadamente norte-americana 5 e do marketing 31, a saber: teoria da atitude, teoria da discrepância, teoria da realização (de expectativas e de necessidades) e teoria da eqüidade. Essas teorias centram-se nos usuários, destacadamente em aspectos psicológicos como crenças, expectativas e percepções.

Vale mencionar que, enquanto alguns modelos teórico-conceituais são bastante desenvolvidos e comentados na literatura, como a teoria da discrepância e a teoria da realização ou confirmação da expectativa, outros são apenas mencionados, como a teoria da eqüidade e a teoria da realização de necessidades.

A teoria da discrepância é a mais utilizada nas pesquisas 28 . Os níveis de satisfação são preditos a partir da diferença entre as expectativas e a percepção da experiência 5,13,28,30,32. Alguns autores buscaram testar esta teoria e observaram que ela é capaz de dar conta de apenas uma parte da variação dos níveis de satisfação 16 .

Na teoria da atitude, a satisfação é entendida como uma atitude, ou seja, uma avaliação positiva ou negativa feita pelo indivíduo sobre um determinado aspecto do serviço ${ }^{5}$. Exemplo desta teoria, o modelo de Linder-Pelz ${ }^{5}$, denominado teoria do valor-expectativa (value-expectancy theory), considera a satisfação como uma atitude positiva relativa à crença de que o cuidado médico contém certos atributos (acesso, eficácia, custo, conveniência etc.) que podem ser avaliados.

A teoria da eqüidade considera que os usuários avaliam os serviços em termos de "ganhos" e "perdas" individuais e na comparação com outros usuários. Embora pouco referida pelos estudos empíricos, a teoria da eqüidade tem sido bastante valorizada pelos estudiosos do tema, pois postula que o usuário avalia os serviços segundo critérios de eqüidade 5 e, dessa forma, introduz elementos de comparação social, confrontando a avaliação de um único paciente com as dos demais 28,33 .

Na teoria da realização, a satisfação é dada simplesmente pela diferença entre o que é desejado, ou esperado, e o que é obtido 5,28. Esta teoria tem sido bastante criticada por não considerar a experiência do serviço. Dois tipos de teoria da realização podem ser encontrados: a realização da expectativa e a realização da necessidade. Neste último, estudos mostram que aspectos da efetividade do serviço e o nível de saúde do usuário são levados em conta nas respostas sobre a satisfação 16 . Kane et al. 34 estudaram a relação entre satisfação e resultados clínicos e encontraram que, embora relacionados, outros fatores atuaram na determinação da satisfação.

A teoria da realização da expectativa ( $f u l$ fillment expectation) ou teoria da confirmação da expectativa (disconfirmation expectancy theory) é também bastante utilizada nos estudos empíricos, sendo, sem dúvida, a mais criticada pela literatura. De acordo com este modelo, o usuário demonstra satisfação quando recebe aquilo que esperava do serviço ou mais do que esperava $5,18,28,32$.

O problema do emprego desta teoria para a avaliação da qualidade dos serviços está em que este indicador permite somente saber o quanto um serviço foi melhor que a expectativa do respondente. Corre-se o risco de não se avaliarem os serviços de saúde, mas algo completamente diferente, baseado apenas no desejo de alguém 16,28. Alguns autores consideram que essa associação é própria para o senso comum, mas não cabe para fundamentar a prática da pesquisa em saúde $28,35,36$.

Com efeito, Newsome \& Wright 23 (p. 162) consideram que a percepção da qualidade antecede a satisfação do paciente, podendo ocorrer independente da experiência relativa a um 
serviço, como em: “Eu sei que Dr. Xfaz um trabalho de alta qualidade, embora eu não tenha sido seu paciente". No entanto, para estes mesmos autores, a satisfação só ocorre em decorrência da experiência atual com o serviço em questão: "Eu não posso te dizer o quanto estou satisfeito com Dr. X, porque eu nunca fui tratado por ele".

Ademais, estudos têm buscado testar a correlação entre satisfação e expectativas e, enquanto alguns apontam que essa associação é inconsistente $7,24,37,38$, outros refutam a sua existência 16. Neste último caso, é possível que haja satisfação para um dado item do serviço, em relação ao qual o paciente não tinha nenhuma expectativa 39 . Vale destacar que essa variabilidade de correlações reportadas na literatura entre satisfação e expectativas pode ser explicada pela diversidade de metodologias utilizadas nos estudos e pelas diferentes dimensões dos serviços avaliadas 24 .

Outros autores, por sua vez, defendem o emprego da categoria expectativa na avaliação da satisfação. Staniszewska \& Ahmed 24 mostram que esta é uma medida mais complexa do que a literatura costuma retratar. Pode variar em tipos, em valores (positivas e negativas) e modificam-se ao longo do cuidado recebido. Para estes autores, as expectativas negativas, por exemplo, são importantes de serem estudadas na medida em que podem refletir "a consciência política e econômica do paciente sobre as circunstâncias em que os serviços se encontram" 24 (p. 371), como em situações em que o atendimento é reconhecido como de baixa qualidade, mas porque existem muitos pacientes para serem atendidos. A formação de expectativas negativas também pode afetar os resultados do tratamento, uma vez que os estudos sobre eficácia de placebos mostram que estes estão associados às expectativas positivas do cuidado 24 .

Newsome \& Wright 23 citam o modelo de Zeithaml \& Bitner, que diferencia os níveis de expectativas dos usuários. O primeiro nível é dos serviços desejados (desired services), aquele que o usuário gostaria de receber. Os usuários, no entanto, reconhecem que nem sempre é possível alcançar o serviço desejado, portanto eles carregam um segundo nível de expectativas, os serviços adequados (adequate services). Este representa um mínimo tolerável do desempenho dos serviços. Finalmente, os serviços presumidos (predicted services) representam o nível de expectativas no qual os usuários dimensionam aquilo que de fato os aguarda nos serviços, fazendo inferências sobre a probabilidade do desempenho dos serviços. Com isso, observa-se que os usuários distinguem antecipadamente diferentes tipos de desempenho dos serviços e estabelecem uma zona de tolerância, situada entre os serviços desejados e os adequados. Quando o desempenho é superior ou inferior à zona de tolerância, o usuário manifesta satisfação/insatisfação.

Embora este modelo contribua trazendo os diferentes níveis de expectativa dos usuários, é também um modelo centrado somente na realização da expectativa destes. De modo semelhante, Pascoe 31, com base em uma vasta revisão de literatura, formula seu próprio modelo conceitual de satisfação. Considera que a satisfação é uma avaliação pessoal do serviço recebido, baseada em padrões subjetivos de ordem cognitiva e afetiva e é estabelecida pela comparação entre a experiência vivida e critérios subjetivos do usuário. Esses critérios incluem a combinação dos seguintes elementos: um ideal de serviço, uma noção de serviço merecido, uma média da experiência passada em situações de serviços similares e um nível subjetivo mínimo da qualidade de serviços a alcançar para ser aceitável 31. Segundo Aharony \& Strasser 6, o modelo de Pascoe também não foi testado ou comprovado, apesar de ser bastante empregado na literatura.

Cabe ressaltar que autores que têm revisado a literatura sobre expectativas dos usuários 40,41 consideram que existe pouco arsenal teórico e metodológico na literatura disponível para o estudo da expectativa do paciente e apontam que o conceito de expectativa, as tipologias utilizadas e os métodos de pesquisa precisam ser mais bem investigados. Marcinowicz 40 sugere a utilização de pesquisas qualitativas e a necessidade de padronização da terminologia sobre expectativa dos usuários na literatura internacional.

Ainda que os enfoques teóricos descritos até agora se superponham em alguma medida, alguns podem ser caracterizados como "modelos combinados". Swan 42 propõe um modelo que contém quatro construtos básicos: (i) a percepção do desempenho do serviço, (ii) a confirmação da expectativa a respeito do desempenho e a percepção de tratamento eqüitativo, (iii) a satisfação geral e (iv) a intenção de revisitar ou evitar o serviço no futuro. A contribuição do modelo de Swan 42 , denominado de quatro estágios, é que ele leva em consideração a eqüidade - o sentimento do paciente de ter sido tratado justa ou injustamente - como parte importante do processo de avaliação, além da expectativa e da percepção do desempenho do serviço. Esses fatores estariam relacionados com a satisfação geral, que, por sua vez, determina o retorno ao serviço. 
Com efeito, a satisfação tem sido estudada como uma variável causal que leva o usuário à procura do atendimento médico e, ao mesmo tempo, um resultado desta experiência 5,12,31, 43,44. Para Wartman et al. ${ }^{44}$, no primeiro caso, admite-se que deve haver uma satisfação geral com o sistema de saúde ou uma crença em sua efetividade (garantia de utilização). No segundo caso, quando a satisfação determina o retorno do usuário, há uma satisfação mais específica com o serviço em questão (garantia de retorno). De modo análogo, o modelo de dupla causalidade entre satisfação e uso de serviços, elaborado por Zastony et al. 12, distingue a satisfação como "input" e "output".

Os modelos explicativos anteriormente referidos refletem a introdução de paradigmas do marketing no campo da saúde. Tal qual a busca da fidelidade do consumidor a produtos e serviços no mercado, a satisfação aqui é estudada em associação ao conhecimento das intenções de comportamento do usuário, buscando monitorar e prever o retorno aos serviços ou a procura de outros provedores. Sem minimizar tal preocupação, essas questões de fato interessam ao campo da saúde, uma vez que há ganhos valiosos quando da continuidade do tratamento, podendo este interferir na efetividade terapêutica, com a maior adesão aos procedimentos recomendados.

\section{Determinantes da satisfação de usuários: fatores relativos aos serviços e aos usuários}

A despeito das dificuldades em definir a satisfação, muitos estudos buscaram estudar a relação entre a satisfação e as variáveis que a determinam. Apesar do esforço dessas investigações, ainda se sabe pouco sobre a natureza ou o número de fatores que influenciam a satisfação 45,46. Os resultados encontrados na literatura são divergentes, indicando baixa consistência entre as pesquisas.

O trabalho de Weiss 11 sobre os determinantes da satisfação tem sido bastante citado. Weiss, após uma revisão extensa de literatura sobre o tema, apresenta quatro grupos principais de determinantes da satisfação: (i) características dos pacientes (incluindo as sócio-demográficas; as expectativas destes sobre a consulta médica e os seus estados de saúde); (ii) características dos profissionais que prestam o atendimento (incluindo traços de personalidade, qualidade técnica e a "arte do cuidado"); (iii) aspectos da relação médico-paciente; (iv) fatores estruturais e ambientais (incluindo o aces- so, forma de pagamento, tempo de tratamento, marcação de consulta e outros).

De modo geral, destacam-se dois grandes grupos de fatores relacionados com a satisfação. Um relativo ao serviço e aos seus provedores, e outro inerente aos próprios usuários. A literatura diverge bastante no que se refere aos determinantes relativos aos usuários. Enquanto uns estudos mostram associação positiva, outros mostram associação negativa, havendo ainda um terceiro grupo, que não encontra associação alguma 32,45 .

A variável que apresenta maior concordância entre os estudos é a idade 16,33. Segundo Aspinal et al. 16, é possível que tal explicação não esteja relacionada com a idade per se, mas com as características de grupos mais idosos, como o maior carisma ou menores expectativas. Para a variável gênero, embora alguns autores não encontrem variação, outros apontam que as mulheres estão mais aptas a criticar os serviços, constituindo-se em boas informantes para as pesquisas 17. Mesmo que alguns autores na literatura internacional não encontrem associação entre satisfação e renda, ou entre satisfação e escolaridade, no Brasil parece haver uma correlação inversa, estando os usuários de camadas mais populares mais satisfeitos com os serviços 15,33.

Com relação aos determinantes relativos aos serviços, também é observada divergência. A maioria dos estudos atribui a satisfação a aspectos humanitários da relação médico-paciente, à quantidade e qualidade das informações recebidas, à efetividade dos problemas e à continuidade do atendimento 6,26. No caso de pesquisas brasileiras, no entanto, sobretudo em serviços públicos, além dos fatores humanitários, os autores referem que os usuários se satisfazem com o acesso aos serviços e com a disponibilidade de insumos, como vacinas e medicamentos 15 .

O estudo de meta-análise realizado por Hall \& Dornan 47 investigou os aspectos dos serviços estudados em 221 artigos sobre satisfação do usuário, o que permite visualizar quais os fatores dos serviços relacionam-se com a satisfação. Os autores, contudo, fazem a ressalva de que seu trabalho mostra a freqüência com que cada aspecto foi investigado pelo pesquisador, o que não necessariamente representa o quanto o usuário valoriza determinado aspecto do serviço. Neste particular, a revisão de Souza 33 aponta também outros critérios derivados da opinião de usuários: ser tratado com cortesia pela equipe, ser reconhecido por ela, ter enfermeiro disponível na unidade, haver disponibilidade de consulta em 48 horas, tempo de es- 
pera da consulta ser inferior a vinte minutos, unidade não ser muito grande, ser possível a execução de exames na unidade. No caso de hospitais, ressalta-se ainda a alimentação, informação, conforto, qualidade dos equipamentos, confiança na equipe, horários de visitas, acessibilidade e privacidade 21,33 .

Sixma et al. 48 buscaram identificar, por intermédio de análise fatorial, a força com que a satisfação se correlaciona com as variáveis relacionadas aos usuários e ao serviço. Encontraram que de $90 \%$ a $95 \%$ das variações da satisfação eram resultados de variáveis relacionadas aos pacientes. Apenas 5\% diziam respeito aos serviços e seus provedores. Diante disso, os autores consideram que a satisfação é uma medida subjetiva, centrada no usuário, e que fatores dos serviços podem ser rechaçados por este tipo de avaliação.

De fato, sendo o conceito de satisfação de natureza eminentemente subjetiva, fatores psicossociais têm ganhado relevância no entendimento da satisfação. Ainda conforme Weiss 11, destaca-se a satisfação com a vida em geral e com a comunidade em que reside, o nível de credibilidade dos médicos da região, sentimentos elevados de controle interno (o indivíduo considera-se responsável pela própria vida), e não de controle externo (o indivíduo imagina a vida controlada por fatores externos à sua pessoa), e o estado atual da saúde do usuário, que pode intervir não só pela gravidade do quadro, mas pela limitação física, psicológica, social ou mental decorrente da doença, como na depressão. A expectativa dos pacientes é, sem dúvida, o determinante mais estudado pela literatura, embora, conforme foi mostrado anteriormente, nem todos os autores concordem com essa associação.

Mais recentemente, estudos de revisão de literatura 49,50 demonstraram a associação existente entre continuidade do cuidado e satisfação do paciente. A revisão crítica de Saultz \& Albedaiwi 49 demonstrou que existe uma alta significativa de satisfação quando da presença do cuidado interpessoal continuado; por sua vez, Cabana \& Jee 50 revelam que existe uma associação consistente entre continuidade do cuidado e satisfação do paciente, sobretudo na atenção a pacientes com condições crônicas, como asma e diabetes.

Em síntese, muitos aspectos são levados em consideração na produção da satisfação ou insatisfação, variando de estudo para estudo. Um melhor entendimento sobre esses determinantes requer pesquisas adicionais que se valham de métodos e técnicas padronizados, visando à devida comparação entre os estudos.

\section{A aplicação do conceito de satisfação de usuários nos serviços de saúde: qual a função das avaliações?}

É comum a referência de que a medida de satisfação de usuários admite distintas funções 30,51. O uso indiscriminado da satisfação para diferentes propósitos pode ser explicado pela fragilidade teórico-conceitual do termo "satisfação" anteriormente mencionada. De modo geral, distinguem-se duas funções: os estudos servem para descrever os serviços na perspectiva dos usuários 24,35,52 ou para avaliar a qualidade dos serviços de saúde 7,8,30,39,53. Esta última é considerada a mais freqüente função da avaliação de satisfação de usuários.

No caso da avaliação da qualidade, de acordo com Donabedian 54,55, a satisfação insere-se no componente relacional entre usuários e profissionais. A avaliação da qualidade dos serviços admite sempre duas dimensões: o desempenho técnico, qual seja, a aplicação do conhecimento e da tecnologia médica a fim de maximizar os benefícios e reduzir os riscos, e o relacionamento com o paciente. Segundo este autor, a avaliação da qualidade do cuidado à saúde pode dar-se em seus três componentes: estrutura (recursos utilizados pelo serviço), processo (procedimentos empregados no manejo dos problemas dos pacientes) e resultado (estado de saúde do paciente ou da comunidade resultante da interação com o serviço de saúde).

A satisfação do usuário geralmente é caracterizada como uma avaliação de resultados, estando associada à efetividade do cuidado ou a um ganho específico de um determinado tipo de intervenção 56,57 , sendo também descrita em termos de saúde psicológica do indivíduo 55. $\mathrm{Na}$ qualidade de processo, refere-se, sobretudo, à relação interpessoal entre profissional de saúde e usuário 18; na avaliação da estrutura dos serviços, a satisfação serve para avaliar o contexto e insumos 31 . Vale destacar que, ainda dentro da avaliação da qualidade, a satisfação de usuário constitui-se em um componente da aceitabilidade social 53; esta aceitabilidade representa a aceitação e aprovação de um serviço de saúde por parte de uma população.

Alguns autores fazem críticas ao emprego da satisfação para a avaliação da qualidade, ao considerar que, uma vez que a satisfação está fortemente baseada nas expectativas dos usuários, podem-se estar avaliando outros itens, e não especificamente a qualidade dos serviços. Quando se busca avaliar a qualidade de um serviço de saúde, é necessário assegurar-se que o serviço, e não outros fatores, determine a sua qualidade 16. 
Observa-se que o conceito de satisfação empregado pode refletir diferentes objetivos de pesquisa (avaliar a qualidade ou conhecer a percepção do usuário) e encontra-se relacionado a eventos médicos e não médicos. A satisfação serve para avaliar desde as "amenidades do cuidado", referindo-se a desde alimentação, limpeza e conforto, até qualidade técnica dos serviços.

\section{Como os usuários avaliam os serviços de saúde?}

A outra função mencionada para os estudos de satisfação de usuários é a de oferecer uma avaliação dos serviços prestados na perspectiva dos usuários. Todavia, tem-se questionado se o emprego do conceito de satisfação dá conta de tal fenômeno 13,16,18,28,35,39,58.

Estudos mostram que a avaliação feita pelo usuário sobre o serviço não é descrita em termos de satisfação, ou seja, os usuários raramente usam a frase "estou satisfeito" para descrever o serviço que receberam 18,28 . Sendo assim, a satisfação não seria a melhor medida para se alcançar a perspectiva do usuário.

Collins \& Nicholson 59 observaram que o termo "satisfação" é bastante complexo e que nem sempre é compreendido pelo usuário. Revela-se um termo vago e fluido, sendo definido, redefinido e reavaliado diversas vezes pelo usuário durante um processo de avaliação dos serviços de saúde. A esse respeito, Williams 28 adverte que é preciso perguntar ao usuário o que ele entende por satisfação, antes de avançar na avaliação pretendida.

Sofaer \& Firminger 60 relatam a dificuldade de identificar questões conceituais e metodológicas relativas à satisfação de usuários, marcadamente a confusão entre a percepção dos pacientes e a satisfação dos pacientes, bem como a dificuldade de determinar se as variações na percepção dos pacientes devem ser atribuídas às diferenças nas expectativas dos pacientes ou às diferenças em suas experiências atuais.

Alguns autores recomendam que as pesquisas devam compreender como o paciente avalia os serviços e estudar os dispositivos cognitivos acionados quando da avaliação de um serviço 28,35,39. Motivados por essa questão, em 1998, Williams et al. 61 desenvolveram um modelo que identifica que os usuários avaliam os serviços de saúde valendo-se da percepção de que o serviço é obrigado a dar conta de seu problema de saúde e, em caso de não-cumprimento dessa obrigação, avaliam se o serviço é culpado pela experiência negativa do usuário.
Este modelo também fornece uma explicação para as altas taxas de satisfação reportadas por diversas pesquisas. Para estes autores, a expressão de satisfação do usuário não necessariamente reflete uma avaliação positiva. Os pacientes sentem-se satisfeitos independentemente da boa qualidade do cuidado recebido e a insatisfação é manifesta apenas em eventos extremamente negativos. Portanto, a satisfação não indica que um cuidado foi bom, mas que não foi "muito ruim" 61.

Por fim, pode-se observar que há um conjunto de críticas aos modelos oferecidos para a satisfação de usuários. Tais modelos são considerados de abordagem funcionalista 62 e mecanicista 13, desprezando questões prioritárias na avaliação dos serviços. Williams 28 , ainda mais incisivo, considera a satisfação como um conceito infundado, surgido no bojo do consumismo e que só faz endossar o status quo dos serviços, contribuindo muito pouco para a avaliação de sua qualidade.

Segundo Carr-Hill 13 (p. 242), as teorias fornecidas são modelos simplistas pautados no seguinte esquema: "Os pacientes chegam nos serviços com objetivos; os médicos fazem algo pelos objetivos (ou não); um 'satissímetro' ('satissometer') registra o resultado". Para este autor, independentemente do conceito de satisfação, os modelos não refletem a relação médico-paciente e os usuários apresentam-se numa atitude sempre passiva em face do serviço. Na opinião de Aspinal et al. 16, não é problematizado se os usuários participam de forma ativa no processo de avaliação de modo a influenciar sua própria satisfação. O usuário insere-se nos modelos sempre numa perspectiva "skinneriana" 6 .

\section{Conclusões}

A despeito do reconhecimento de que todo modelo teórico é sempre parcial e aproximativo, é necessário reconhecer a pertinência das críticas que apontam inconsistências teórico-conceituais importantes no âmbito dos estudos de satisfação de usuários. Os conceitos empregados, além de revelarem-se inconsistentes e de baixa predição, são relacionados de modo vago e difuso a uma série de eventos dos serviços, entre médicos e não médicos. Se a variedade de conceitos formulados para abordar a satisfação de usuário pode evidenciar os esforços em fundamentar teoricamente o tema, revela também as dificuldades, enfrentadas até então, de se estabelecer um referencial teórico estável e consensual. A falta de consenso entre os pesquisadores traduz uma baixa validação externa 
das teorias. Observa-se ainda a falta de consistência interna, uma vez que algumas teorias existentes não foram testadas por seus autores e, quando foram, na maioria dos casos, sofreram refutação.

Ademais, as teorias e conceitos apresentados são oriundos de referenciais teóricos do marketing e da psicologia social, nem sempre reprodutíveis no campo da saúde. Neste particular, chama a atenção a ênfase dada à categoria expectativa, ao passo em que é desprezada a noção de necessidade, uma categoria bastante valorizada no campo da saúde, particularmente no âmbito da saúde coletiva.

Convém problematizar a própria validade do emprego da categoria expectativa na avaliação de satisfação, uma vez que valoriza uma visão idealizada do usuário sobre o serviço. Corre-se o risco, assim, de cair no "psicologismo", além de desprezar aspectos importantes do serviço em questão. Os modelos da discrepân- cia, por seu turno, ainda que assumam a expectativa, permitem uma avaliação da experiência do usuário no que se refere ao serviço, estando mais voltados para a "realidade" dos serviços.

A análise da literatura permite inferir também que há perda da utilidade analítica do conceito de satisfação, na medida em que ele se aplica a diversos aspectos dos serviços, desde as amenidades destes à dimensão técnica, assumindo distintas funções.

Por fim, a constituição de um consenso de especialistas deve ser encorajada, a fim de buscar uma validação para o conceito de satisfação de usuários e uma operacionalização para a sua medida. Particularmente, pensamos que a avaliação de satisfação de usuários em saúde deve ser valorizada, como parte dos estudos das relações interpessoais no âmbito dos serviços de saúde e como instrumento de controle social.

\section{Resumo}

O presente artigo desenvolve uma análise crítica da produção científica sobre o tema da satisfação de usuários de serviços de saúde, focalizando, especificamente, os aspectos teórico-conceituais. Abrange a discussão acerca da compreensão do conceito de satisfação e seus quadros teóricos de referência, dos determinantes da satisfação e a aplicação do conceito no campo dos serviços. Foram analisados 56 artigos publicados especialmente em revistas indexadas pelo MEDLINE e Web of Science no periodo de 1970 a 2005. Os conceitos encontrados provêm principalmente do marketing e da psicologia social, destacando-se a teoria da atitude, da realização da expectativa ou necessidade e da eqüidade. Nota-se que há uma perda da utilidade analítica do conceito satisfação na medida em que seu conceito é ampliado para diversas dimensões dos serviços de saúde.

Satisfação dos Consumidores; Avaliação dos Serviços; Qualidade

\section{Colaboradores}

M. A. Esperidião participou de todas as etapas do desenvolvimento da pesquisa e do artigo. L. A. B. Trad participou da elaboração do artigo, análise e revisão do texto.

\section{Agradecimentos}

Agência financiadora: Coordenação de Aperfeiçoamento de Pessoal de Nível Superior (CAPES). 


\section{Referências}

1. Chen TH. Theory-driven evaluations. Beverly Hills: Sage; 1990.

2. Deslandes SF. Concepções em pesquisa social: articulações com o campo da avaliação em serviços de saúde. Cad Saúde Pública 1997; 13:103-7.

3. Mendonça ND. Uma questão de interdisciplinaridade: o uso dos conceitos. 2a Ed. Petrópolis: Editora Vozes; 1985.

4. Turris AS. Unpacking the concept of patient satisfaction: a feminist analysis. J Adv Nurs 2005; 50: 293-8.

5. Linder-Pelz S. Toward a theory of patient satisfaction. Soc Sci Med 1982; 16:577-82.

6. Aharony L, Strasser S. Patient satisfaction: what we know about and what we still need to explore. Med Care Rev 1993; 50:49-79.

7. Baron-Epel O, Dushenat M, Friedman N. Evaluation of the consumer model: relationship between patient's expectations, perception and satisfaction with care. Int J Qual Health Care 2001; 13:317-23.

8. Bernhart MH, Wiadnyana IGP, Wihardjo H, Pohan I. Patient Satisfaction in developing countries. Soc Sci Med 1999; 48:989-96.

9. Johansson P, Oléni M, Fridlund B. Patient satisfaction with nursing care in the context of the health care: a literature study. Scand J Caring Sci 2002; 16:337-44.

10. Ware JE, Snyder MK, Wright WR, Davies AR. Defining and measuring patient satisfaction with medical care. Eval Program Plann 1983; 6:247-63.

11. Weiss GL. Patient satisfaction with primary medical care: evaluation of sociodemographic and predispositional factor. Med Care 1988; 26:383-92.

12. Zastowny TR, Roghmann KJ, Cafferata GL. Patient satisfaction and the use of health services: exploration in causality. Med Care 1989; 27:705-23.

13. Carr-Hill RA. The measurement of patient satisfaction. J Public Health Med 1992; 14:236-49.

14. Gilbert RG, Nicholls JAF, Roslow S. A mensuração da satisfação dos clientes do setor público. Revista do Serviço Público 2000; 51:26-40.

15. Santos MP. Avaliação da qualidade dos serviços públicos de atenção à saúde da criança sob a ótica do usuário [Dissertação de Mestrado]. Salvador: Instituto de Saúde Coletiva, Universidade Federal da Bahia; 1995.

16. Aspinal F, Addington-Hall, Hughes R, Higginson IJ. Using satisfaction to measure the quality of palliative care: a review of the literature. J Adv Nurs 2003; 42:324-39.

17. Coyle J. Exploring the meaning of "dissatisfaction" with health care: the importance of "personal identity threat”. Sociol Health Illn 1999; 21:95-123.

18. Fitzpatrick R, Hopkins A. Problems in the conceptual framework of patient satisfaction research: an empirical exploration. Sociol Health Illn 1983; 5:297-311.

19. Hudak PL, Wright JG. The characteristics of patient satisfaction measures. Spine 2000; 25:3167-77.

20. Jacob G, Bengel J. The construct "patient satisfaction": a critical review. Z Klin Psychol Psychiatr Psychother 2000; 48:280-301.

21. Locker D, Dunt D. Theoretical and methodological issues in sociological studies of consumer sat- isfaction with medical care. Soc Sci Med 1978; 12:283-92.

22. Mahon PY. An analysis of the concept "patient satisfaction" as it relates to contemporary nursing care. J Adv Nurs 1996; 24:1241-8.

23. Newsome PRH, Wright GH. A review of patient satisfaction: 1. Concepts of satisfaction. Br Dent J 1999; 186:161-5.

24. Staniszewska S, Ahmed L. The concepts of expectation and satisfaction: do they capture the ways patients evaluate their care? J Adv Nurs 1999; 29:364-72.

25. Thompson A, Suñol R. Expectations of patient satisfaction - concepts, theory and evidence. Int J Qual Health Care 1995; 7:127-41.

26. Ware JE, Snyder MK. Dimensions of patient attitudes regarding doctors and medical care services. Med Care 1975; 13:669.

27. Weaver M, Patrick DL, Markson LE, Martin D, Frederic I, Berrger M. Issues in the measurement of satisfaction with treatment. Am J Manag Care 1997; 3:579-94.

28. Williams B. Patient satisfaction: a valid concept? Soc Sci Med 1994; 38:509-51.

29. Edwards C, Staniszewska S. Accessing the user's perspective. Health Soc Care Community 2000; 8:417-24.

30. Sitzia J, Wood N. Patient satisfaction: a review of issues and concepts. Soc Sci Med 1997; 45:1829-43.

31. Pascoe GC. Patient satisfaction in primary health care: a literature review and analysis. Eval Program Plann 1983; 6:185-210.

32. Fox JG, Storms DM. A different approach to social demographic predictors for satisfaction with health care. Soc Sci Med 1981; 15:557-67.

33. Souza EM. A satisfação dos idosos com os serviços de saúde: um estudo de prevalência e de fatores associados em Taguatinga, Distrito Federal [Dissertação de Mestrado]. Brasília: Faculdade de Ciências da Saúde, Universidade de Brasília; 1997.

34. Kane RL, Maciejewski M, Fich M. The relationship of patient satisfaction with care and clinical outcomes. Med Care 1997; 35:714-30.

35. Avis M, Bond M, Arthur A. Questioning patient satisfaction: an empirical investigation in two outpatient clinics. J Adv Nurs 1997; 44:85-92.

36. Like R, Zyzanski SJ. Patient satisfaction with the clinical encounter: social psychological determinants. Soc Sci Med 1987; 24:351-7.

37. Kravitz RL. Measuring patients' expectations and request. Ann Intern Med 2001; 134:881-8.

38. Rao JK, Weimberger M, Kroenke K. Visit-specific expectations and patient-centered outcomes - a literature review. Arch Fam Med 2000; 9:1148-55.

39. Owens DJ, Bachelor C. Patient satisfaction and the elderly. Soc Sci Med 1996; 42:1483-91.

40. Marcinowicz L. Patient's expectations for medical care-concepts, typology, research methods. Pol Merkuriusz Lek 2004; 17:95-7.

41. Redman RW, Lynn MR. Assessment of patient expectations for care. Res Theory Nurs Pract 2005; 19:275-85.

42. Swan J. Deepening the understanding of hospitals patient satisfaction: fulfillment and equity ef- 
fects. J Health Care Mark 1985; 5:7-18.

43. Parasuraman A, Zeithaml V, Berry L. A multipleitem scale for measuring customer perceptions of service quality. Journal of Retailing 1988; 64:1240.

44. Wartman AS, Morlock LL, Malitz FE, Palm AE. Patient understanding and satisfaction as predictors of compliance. Med Care 1983; 21:386-91.

45. Lewis JR. Patient views on quality care in general practice: literature review. Soc Sci Med 1994; 39: 655-70.

46. Ross CK, Steward CA, Sinacope JM. The importance of patient preferences in the measurement of health care satisfaction. Med Care 1993; 31:113849.

47. Hall JA, Dornan MC. Meta-analysis of satisfaction with medical care: description of research domain and analysis of overall satisfaction levels. Soc Sci Med 1988; 27:637-44.

48. Sixma HJ, Spreeuwenberg PMM, van der Pasch MAA. Satisfaction with the general practitioner a two level analysis. Med Care 1998; 36:212-29.

49. Saultz JW, Albedaiwi W. Interpersonal continuity of care and patient satisfaction: a critical review. Ann Fam Med 2004; 2:44-51.

50. Cabana MD, Jee SH. Does continuity of care improve patient outcomes? J Fam Pract 2004; 53:97480.

51. Noronha Filho G. O que fazer da literatura sobre satisfação do paciente: uma proposta para os serviços. Revista Portuguesa da Saúde Pública 1990; 8:59-64.

52. Fitzpatrick R. Surveys of patient satisfaction: I important general considerations. BMJ 1991; 302: 1129-32.
53. Donabedian A. The seven pillars of quality. Arch Pathol Lab Med 1990; 114:1115-8.

54. Donabedian A. Exploration in quality assessment and monitoring. v. 1. Ann Arbor: Health Administration Press; 1980.

55. Donabedian A. La calidad de la atención médica - definición y métodos de evaluación. México DF: La Prensa Mexicana; 1984.

56. Oliveira CG. Análise do conceito de satisfação do usuário na obra de Donabedian. Rio de Janeiro: Instituto de Medicina Social, Universidade do Estado do Rio de Janeiro; 1992.

57. Vuori H. Patient satisfaction - an attribute or indicator of the quality of care? QRB Qual Rev Bull 1987; 13:106-8.

58. Calnan M. Towards a conceptual framework of lay evaluation of health care. Soc Sci Med 1988; 27:927-33.

59. Collins K, Nicolson P. The meaning of "satisfaction" for people with dermatological problems: Reassessing approaches to qualitative health psychology research. J Health Psychol 2002; 7:615-29.

60. Sofaer S, Firminger K. Patient perception of the quality of health services. Ann Rev Public Health 2005; 26:51-9.

61. Williams B, Coyle J, Healy D. The meaning of patient satisfaction: an explanation of high reported levels. Soc Sci Med 1998; 47:1351-9.

62. Oliveira CG. Introdução à crítica das noções empregadas em pesquisa sobre "satisfação do usuário”. Saúde Debate 1990, 30:32-4.

Recebido em 13/Abr/2005

Versão final reapresentada em 27/Set/2005 Aprovado em 22/Nov/2005 